\title{
Construction and directional evolution of anti- enrofloxacin ScFv antibody for the immunoassay of fluoroquinolones
}

\section{Guangxu Xing}

Henan Academy of Agricultural Sciences

\section{Yunshang Zhang}

Henan Academy of Agricultural Sciences

\section{Fangyu Wang}

Henan Academy of Agricultural Sciences

\section{Liuding Wen}

Henan Agricultural University

Gaiping Zhang ( $\nabla$ sprinkle.w@126.com )

Henan Academy of Agricultural Sciences https://orcid.org/0000-0002-4791-5916

\section{Research Article}

Keywords: ScFv, Enrofloxacin, recognition mechanism, directional mutagenesis, IC-ELISA

Posted Date: March 16th, 2021

DOI: https://doi.org/10.21203/rs.3.rs-310107/v1

License: (c) (1) This work is licensed under a Creative Commons Attribution 4.0 International License. Read Full License 


\section{Abstract}

A recombinant anti-enrofloxacin single-chain antibody (scFv) was produced for the detection of enrofloxacin. An immunized mouse phage display scFv library with a capacity of $2.35 \times 10^{9} \mathrm{CFU} / \mathrm{mL}$ was constructed and used for anti-enrofloxacin scFv screening. After four rounds of bio-panning, 10 positives were isolated and identified successfully. The highest positive scFv was expressed in E. coli BL21. Then, its recognition mechanisms were studied using the molecular docking method. The result showed the amino acid residues Leu121 were the key residues for the binding of ScFv to ENR. Based on the results of virtual mutation, the ScFv antibody was evolved by directional mutagenesis of contact amino acid residue Leu121 to Asn. After the expression and purification, an indirect competitive enzyme-linked immunosorbent assay (IC-ELISA) based on the parental and mutant ScFv were established for enrofloxacin respectively. The $\mathrm{IC}_{50}$ value of the assay established with the ScFv mutant was $1.63 \mathrm{ng} / \mathrm{mL}$, while the parental ScFv was $21.08 \mathrm{ng} / \mathrm{mL}$, this result showed highly increased affinity with up to 12.9 folds improved sensitivity. The mean recovery for ENR ranged from $71.80 \%$ to $117.35 \%$ with $10.46 \%$ relative standard deviation between the intra-assay and the inter-assay. The results indicate that we have obtained a highly sensitive anti-ENR scFv by the phage library construction and directional evolution, and the scFv-based IC-ELISA is suitable for the detection of ENR residue in animal derived edible tissues and milk.

\section{Introduction}

Enrofloxacin (ENR) is a synthetic third-generation fluoroquinolones (FQs) antibiotic developed for veterinary treatment. It shows excellent bioavailability and tolerance, long half-life, and good tissue permeability and has bactericidal effect on Gram-negative bacteria, bacteriostatic effect on Gram-positive bacteria (Dalhoff, 2015), and antifungal and antiviral activities (Kang et al., 2019; Lin et al., 2018). However, unreasonable or careless use of antibiotics can lead to residual antibiotics in products of animal origin, can cause bacterial resistance (J. Li et al., 2017; Xu, Wang, Yang, \& Lu, 2013), and damages multiple systems in the body, such as the endocrine, digestive, nervous, and reproductive, and urinary systems (Bird, Etminan, Brophy, Hartzema, \& Delaney, 2013; Patel \& Goldman, 2016). Therefore, to prevent the accumulation of antimicrobial residues that pose a threat to public health, the European Union, the Joint FAO/WHO Expert Committee on Food Additives and China have set maximum residue limits (MRL) of ENR in edible food (e.g.100 $\mu \mathrm{g} / \mathrm{kg}$ in milk and meat).

Given the widespread spread of antibiotics in the environment and food chain, their adverse effects on the ecological environment and human health have received considerable attention (Kumari \& Kumar, 2020). Enzyme-linked immunosorbent assay (ELISA), which is based on the principle that antibodies specifically bind to antigens, is considered the most reliable method for detecting antibodies (Abdelwahab, Loa, Wu, \& Lin, 2015; Cui et al., 2016). In previous studies, researchers have developed ICELISA based on monoclonal antibodies (MAbs) for fluoroquinolone (Fan et al., 2012; Huang et al., 2010; H.t. Zhang et al., 2011). These types of ELISA have detection limits that satisfy the MRI set by the European Union. Although ELISA is a mature and widely used method, it has many rigorous programs for 
preparing traditional antibodies (PAbs and MAbs) from antigen-immunized animals (C. Li et al., 2019). Hence, a simple, rapid, and effective technology for preparing novel antibodies must be developed.

The development of gene engineering techniques facilitated the production of various gene recombinant antibodies, and single-chain variable fragment $(\mathrm{ScFv})$ is the most popular format of recombinant antibody that has been successfully constructed by assembling the variable-heavy (VH) region and light chain (VL) domain of an antibody with a flexible linker (Makvandi-Nejad, Sheedy, Veldhuis, Richard, \& Hall, 2010). The intrinsic properties of ScFv antibodies can be improved by various mutagenesis techniques (Norihiro, 2008). The recognition property of an ScFv antibody can be evolved in vitro (Norihiro, 2010). For the evolution of a ScFv antibody, its recognition mechanism should be studied first, and binding sites, contact amino acids, and intermolecular forces should be determined (Jing et al., 2016). In recent years, molecular docking has been used in analyzing the interactions between ligands and ScFv antibodies, and random mutagenesis and site-directed mutagenesis have been used in obtaining ScFv mutants (Tao et al., 2013; Wen et al., 2012). For example, a recombinant anti-sarafloxacin ScFv antibody can be produced and then evolved through site-directed mutagenesis. The resulting ScFv mutant, which shows constant sensitivity to nine FQs, exhibits sensitivity that is sevenfold the sensitivities of other three analogs. This ScFv mutant has been used as a detection reagent for determining FQ residues in milk (Wang et al., 2016).

In this study, to obtain a highly sensitive anti-ENR ScFv, we constructed a large-capacity and diverse immunized mouse phage display ScFv library for anti-ENR phage ScFv particle screening. Then, we transfected the phage to $E$. coli $\mathrm{BL} 21$ for expression, studied the recognition mechanism through molecular docking methods, and evolved ScFv through directed mutagenesis. IC-ELISA was used in detecting ENR spikes in the samples and evaluate the evolution efficiency of ScFv.

\section{Materials And Methods}

\section{Reagents and Chemicals}

Enrofloxacin (ENR), sarafloxacin (SAR), difloxacin (DIF), ciprofloxacin (CIP), Lomefloxacin (LOM), enofloxacin (ENO), norfloxacin (NOR) and pefloxacin (PEF) were obtained from the China Institute of Veterinary Drug Control (Beijing, China). All chemicals and reagents used in this study were at least analytical grade or better. The standard stock solutions of these FQs were prepared with methanol (10 $\mu \mathrm{g} / \mathrm{mL})$, and their working solutions with series concentrations $(0.1-200 \mathrm{ng} / \mathrm{mL})$ were diluted from the stock solutions with PBS. All the standard solutions were stored at $4{ }^{\circ} \mathrm{C}$ to be stable for 8 weeks. Freund's complete adjuvant (FCA) and Freund's incomplete adjuvant (FIA) were from Sigma (St. Louis, MO, USA). PBS ( $\mathrm{pH}$ 7.2) was prepared by dissolving $0.2 \mathrm{~g} \mathrm{KH}_{2} \mathrm{PO}_{4}, 0.2 \mathrm{~g} \mathrm{KCl}_{1} 1.15 \mathrm{~g} \mathrm{Na}_{2} \mathrm{HPO}_{4}$, and $8.0 \mathrm{~g} \mathrm{NaCl}$ in $1000 \mathrm{~mL}$ deionized water. Washing buffer (PBST) was PBS buffer containing $0.05 \%$ Tween. Coating buffer was carbonate buffer $(0.1 \mathrm{M}, \mathrm{pH}$ 9.6). Substrate buffer was $0.1 \mathrm{M}$ citrate $(\mathrm{pH}$ 5.5). The substrate system was prepared by adding $200 \mu \mathrm{L} 1 \%(\mathrm{w} / \mathrm{v}) \mathrm{TMB}$ in DMSO and $64 \mu \mathrm{L} 0.75 \%(\mathrm{w} / \mathrm{v}) \mathrm{H}_{2} \mathrm{O}_{2}$ into $20 \mathrm{~mL}$ substrate buffer. 
All the restriction enzymes and DNA modification enzymes were molecular biology grade. RNase prep pure Cell /Bacteria Kit was from Tiangen Biotech Co. Ltd (Beijing, China). Prime script RT-PCR Kit, IPTG (isopropyi- $\beta$-D-thirgalactopyranoside), X-Gal, pCANTAB5E Vector Cloning kit, horseradish peroxidaselabeled goat anti-His-tag antibody, restriction enzymes (Sfi / and Not I) and T4 DNA Ligase were from Takara Company (Dalian, China). EasyPure Quick Gel Extraction Kit, EasyPure Plasmid MiniPrep Kit, express vector Pet-32a competent cell Rosetta-gami(DE3), Fast MultiSite Mutagenesis System and LuriaBertani culture medium (liquid and solid) were from TransGen Biotech (Beijing, China). DNA Purification Kit and SDS-PAGE gel preparation kit were from Beijing ComWin Biotech Co. Ltd (Beijing, China). The synthesis of primers and the analysis of gene sequence were performed at sangon Biotechnology Co. Ltd (Shanghai, China).

\section{Immunization}

The expression of anti-ENR MAbs in the spleens of six Balb/c female mice (8 weeks) was induced by immunizing the mice with five rounds of subcutaneous injection of ENR-BSA conjugates. In the first round of immunization, $250 \mu \mathrm{g}$ of ENR-BSA with Freund's complete adjuvant was emulsified for subcutaneous multipoint injection, then four booster injections with Freund's incomplete adjuvant were given at 2 week intervals. The dose of ENR-BSA in PBS was $125 \mu \mathrm{g}$. Antisera were collected 1 week after the third and fourth immunization, and the antibody titer was determined through indirect ELISA. A week after the fourth round of immunization, booster immunization with $150 \mu \mathrm{g}$ of ENR-BSA in PBS was performed. After 5 days, blood and spleen samples were collected for the construction of phage display ScFv library.

\section{Phage display ScFv library construction}

Total RNA was extracted from mouse tissues with TRIzol reagent according to the manufacturer's instructions. Then, total RNA was used as a template in the reverse-transcription of CDNA. The sequences of the primers were used in the amplification of the cDNAs of VH and VL genes for ScFv construction.

The primers used for the amplification of ScFv-coding sequences were designed according to Table 1 and then spliced to a whole ScFv gene through splicing overlap extension PCR (SOE-PCR). The system conditions were as follows: $94{ }^{\circ} \mathrm{C}$ for 5 min, 30 cycles at $94{ }^{\circ} \mathrm{C}$ for $45 \mathrm{~s}, 58^{\circ} \mathrm{C}$ for $60 \mathrm{~s}$, and $72{ }^{\circ} \mathrm{C}$ for $45 \mathrm{~s}$, and final extension at $72{ }^{\circ} \mathrm{C}$ for $10 \mathrm{~min}$. Gene fragments encoding VH and VL were amplified and spliced to a single gene by using a DNA linker encoding a pentadeca peptide (Gly4Ser) ${ }_{3}$ through primary PCR. The system conditions were $94^{\circ} \mathrm{C}$ for $5 \mathrm{~min}, 30$ cycles of at $94^{\circ}$ a for $45 \mathrm{~s}, 60^{\circ} \mathrm{C}$ for $60 \mathrm{~s}, 72{ }^{\circ} \mathrm{C}$ for $45 \mathrm{~s}$, and final extension at $72^{\circ} \mathrm{C}$ for $10 \mathrm{~min}$. PCR products were verified through agarose gel electrophoresis, and the relevant fragments were sequenced. The gene fragments were then digested with $\mathrm{Sfi} /$ and $\mathrm{Not} I$ restriction endonuclease and ligated into PCANTAB5E phagemid vectors. The recombinant vectors were then transformed into E. coli TG1 cells. Serial dilutions of $10^{-1}-10^{-8}$ were plated onto SOB plates $(2 \%$ tryptone, $0.5 \%$ yeast extract, $0.05 \% \mathrm{NaCl}, 2.5 \mathrm{mM} \mathrm{KCl}, 10 \mathrm{mM} \mathrm{MgCl}_{2}$, and $1.5 \%$ Agar powder) that contain $100 \mu \mathrm{g} / \mathrm{mL}$ Amp and $2 \%$ Glu. After inoculation, all the plates were incubated overnight in a previously set incubator at $30^{\circ} \mathrm{C}$, then clones were randomly selected and screened for inserts by 
performing another round of PCR. Finally, the colonies were scraped into $20 \mathrm{~mL}$ of $2 \mathrm{YT}$ (1.6\% Tryptone, 1 $\%$ yeast Extract, and $0.5 \% \mathrm{NaCl}$ ), named the original antibody library, and stored at $-80{ }^{\circ} \mathrm{C}$ in $20 \%$ glycerol.

\section{Phage ScFv particle enrichment and screening}

The phage library underwent four rounds of biopanning with coat antigen ENR-BSA for anti-ENR phage ScFv particle enrichment. A sterile cell flask was coated with $2 \mathrm{~mL}$ of ENR-BSA (the first round was 50 $\mu \mathrm{g} / \mathrm{mL}$, and the remaining three rounds were 25,12 , and $6 \mu \mathrm{g} / \mathrm{mL}$ ) in PBS solution at $4{ }^{\circ} \mathrm{C}$ and left to stand overnight. The flask was washed five times with PBST solution (contains $0.1 \%$ Tween in PBS) and blocked with $5 \%$ MPBS ( $5 \%$ Skim milk powder in PBS) at $37^{\circ} \mathrm{C}$ for $2 \mathrm{~h}$. After being washed with PBST solution, $1 \mathrm{~mL}$ of library phage particles was added into a flask for shaking of $1 \mathrm{~h}$ at $150 \mathrm{rpm}$ at RM, then left to stand for $1 \mathrm{~h}$. The ENR-BSA-bound phage ScFv particles were washed with PBST solution and eluted with $1 \mathrm{~mL}$ of trypsin solution ( $1 \mathrm{mg} / \mathrm{mL}$ in PBS). The eluent was the first round of enrichment library, and the phage ScFv particles were amplified for the next round of enrichment. Four rounds of biopanning were performed. The fourth round of enriched anti-ENR phage particles were infected to $E$. coli TG1 and spread on a TYE-AG medium (contains $100 \mu \mathrm{g} / \mathrm{mL}$ Amp and 1\% Glu) for culturing overnight at $37^{\circ} \mathrm{C}$. Individual colonies were randomly picked and grown in 2xTY-AG medium glucose with 100 $\mu \mathrm{g} / \mathrm{mL}$ ampicillin for $16 \mathrm{~h}$ at $37^{\circ} \mathrm{C}$ at $200 \mathrm{rpm}$. The next day, $10 \mu \mathrm{L}$ of culture per well was transferred into another fresh 96-well plate for culturing for $2 \mathrm{~h}$ at $37^{\circ} \mathrm{C}$ and $200 \mathrm{rpm}$, and $\mathrm{M} 13 \mathrm{KO} 7$ helper phages were added for rescuing $2 \mathrm{~h}$ at $37^{\circ} \mathrm{C}$ and $200 \mathrm{rpm}$. The plate was centrifuged at $3300 \mathrm{rpm}$ for $20 \mathrm{~min}$ at $37^{\circ} \mathrm{C}$, and the pellets were resuspended with $250 \mu \mathrm{L} /$ well of $2 \times$ TY-AK medium and cultured overnight at $30^{\circ} \mathrm{C}$ and $200 \mathrm{rpm}$. Finally, the plate was centrifuged at $4{ }^{\circ} \mathrm{C}$ and $3300 \mathrm{rpm}$ for $30 \mathrm{~min}$, then the supernatant was used in monoclonal phage ELISA for ENR.

\section{Colony PCR and sequencing}

The positive phage ScFv colonies were cultured in a 2xTY-AG medium until the logarithmic phase for colony PCR, and the PCR products were examined by $1 \%$ agarose gel electrophoresis. Selected positive monoclonal phages were sequenced by Sangon Biotechnology (Shanghai, China) Co., Ltd.

\section{Expression and purification of anti-ENR ScFv}

The target gene and prokaryotic expression vector pET-32a were digested with Ncoll and Not/ restriction enzymes and linked using T4 DNA ligase. Then, the positive recombinant plasmid was used in producing E. coli strain BL21 (DE3) and cultured in a Luria-Bertani (LB) medium ( $1 \%$ tryptone, $0.5 \%$ yeast extract, and $1 \% \mathrm{NaCl}$ ) containing $100 \mu \mathrm{g} / \mathrm{mL}$ kanamycin at $37^{\circ} \mathrm{C}$. After the $\mathrm{OD}_{600}$ of the bacterium solution reached $0.6-0.8,1 \mathrm{mM}$ IPTG was added to the culture to induce the expression of ScFv. The culture was further grown at $37^{\circ} \mathrm{C}$ for $16 \mathrm{~h}$. The supernatant was collected and concentrated 100 -fold by using MWCO: 8000-14000 Da of dialysis bag in PEG/ $\mathrm{NaCl}$. The collected pellets were resuspended with PBS for the production of a periplasmic lysate and lysed through sonication for the production of the whole cell lysate. The supernatant and periplasmic and whole cell lysates were used in analyzing the solubility 
of the proteins through SDS-PAGE. Finally, anti-ENR ScFv proteins were purified using Beaver Beads ${ }^{\text {TM }}$ His- $^{-}$ tag Protein Purification magnetic beads.

\section{Denaturation and renaturation of the ScFv protein}

The inclusion bodies were washed five times with PBS containing $0.1 \%$ TritonX-100 and $2 \mathrm{~mol} / \mathrm{mL}$ urea at $2 \mathrm{~h}$ intervals, then the inclusion bodies were solubilized in $20 \mathrm{~mL}$ of $8 \mathrm{~mol} / \mathrm{mL}$ urea solutions and slowly stirring at $4{ }^{\circ} \mathrm{C}$ for $16 \mathrm{~h}$. The solubilized solution was centrifuged at $12000 \mathrm{rpm}$ for $20 \mathrm{~min}$ for the removal of insoluble ingredients. Finally, solution containing denatured ScFv was dialyzed in PBS at $4^{\circ} \mathrm{C}$ for $48 \mathrm{~h}$ for the removal of urea from the protein solutions.

\section{Characterization of ScFv antibody}

Western blot. A volume of ScFv solution was added to a nitrocellulose membrane immersed in blocking buffer (4 \% BSA in PBS [w/v]) for $1 \mathrm{~h}$. Then, a volume of horseradish peroxidase-labeled anti-His-tag antibody (1:2000) was added to the block point, and the membrane was incubated for $2 \mathrm{~h}$ at room temperature. Finally, a volume of substrate solution (4-chloro-1-naphthol) was added for the visualization of the result.

Indirect competitive ELISA. The purified anti-ENR ScFv was used in establishing IC-ELISA. Briefly, $100 \mu \mathrm{L}$ per well of ENR-OVA solution was coated into 96-well plates overnight at $4{ }^{\circ} \mathrm{C}$; then the plates were washed with PBST solution and blocked with $300 \mu \mathrm{L}$ per well of 5\% PBSM (5\% Skim milk powder in PBS) at $37^{\circ} \mathrm{C}$ for $1 \mathrm{~h}$. ScFv $(100 \mu \mathrm{L} /$ well) previously diluted with PBS and a series of ENR standard concentrations $(200,100,80,50,20,10,5,2,1$, and $0.1 \mathrm{ng} / \mathrm{mL})$ were washed with PBST and then mixed. The plates were incubated at $37^{\circ} \mathrm{C}$ for $1 \mathrm{~h}$, then washed with PBST. Avidin conjugated with horseradish peroxidase ( $100 \mu \mathrm{L} /$ well; $1 / 2000$ dilution in PBS) was added to the wells and incubated at $37^{\circ} \mathrm{C}$ for 30 min. The wells were then washed five times with PBST, and $100 \mu \mathrm{L} /$ well of TMB substrate was added and incubated for $10 \mathrm{~min}$ in the dark at room temperature. Color reaction was stopped with the addition of sulfuric acid ( $2 \mathrm{M}, 50 \mu \mathrm{L} /$ well). Finally, absorbance was measured at $450 \mathrm{~nm}$ with an automatic microplate reader (Thermo, USA). The $\mathrm{IC}_{50}$ value, assay dynamic range, and limit of detection (LOD) served as the criteria for evaluating IC-ELISA. The inhibition ratios of anti-ENR ScFv, IC10, IC20, IC 50 , and IC80 were calculated using the formula $[(P-S-N)] /(P-N)] \times 100 \%$, where $P$ is the OD450 value of the positive sample (50 $\mu \mathrm{L}$ of anti-ENR ScFv mixed with $50 \mu \mathrm{L}$ of CBS), $S$ is the OD450 value of the standard ( $50 \mu \mathrm{L}$ of ScFv mixed with $50 \mu \mathrm{L}$ of the serial concentration of ENR), and $\mathrm{N}$ is the OD450 value of the negative control ( $100 \mu \mathrm{L}$ of CBS).

\section{Homology modeling and molecular docking}

In this experiment, the possible template sequences of the anti-ENR ScFv model were searched in the NCBI database (https://www.ncbi.nlm.nih.gov/), and sequence comparison was performed in the BLAST section for selection template of a high consistency with the anti-ENR ScFv model. The sequence with a high score and low e-value was used as a template sequence for anti-ENR ScFv model building. Then, the 
SWISS MODEL online server was used in the homology modeling of anti-ENR ScFv. To verify the reliability of the homology modeling results and determine the best model structure, we used Procheck, Verify3D, and ERRAT programs in the evaluation of the consistency of the constructed anti-ENR ScFv homology model and selected the best receptor model for further molecular docking study. To study the binding mode of ENR with anti-ENR-ScFv and find key residues, we used MOE 2015.10 in exploring the molecular docking of ENR with anti-ENR-ScFv. In the Dock module, ENR was docked into the active site of anti-ENR-ScFv through the method of Induced Fit and under Amber10: EHT forcefield. The docking ligand, which had 30 docking conformations after default parameters were used, were used for further analysis.

\section{Directional mutagenesis of ScFv antibody}

The binding affinity of ENR with ScFv-ENR antibody was improved through the virtual mutation of the potential key residues of ScFv-ENR. The process was based on the study of binding mode of anti ENR ScFv with ENR. MOE 2015.10 software was used in conducting the virtual mutation of amino acid residues that affect the binding of ENR with ScFv and directly replace them with other amino acids. In this study, Asn was used to replace Leu121 for the production of the mutant of ScFv-ENR antibody. Then, the structure of the virtual mutation ScFv model was optimized, and a stable ScFv mutation model was obtained. Subsequently, the docking study of ENR with ScFv mutation was performed through the method of Induced Fit, and 30 conformations were obtained using default parameters. During the experiments, the ScFv gene in the express vector ScFv-pCANTAB5E was mutated directly for the production of a mutated express vector with a fast-multisite mutagenesis system according to the manufacturer's recommended protocol. Then, the mutated express vector was expressed for the production of an ScFv mutant using the procedures described above. ScFv mutants were identified and analyzed through SDS-Page and IC-ELISA.

\section{Sample Preparation and Cross-reactivity analysis}

Beef, pork, milk, and chicken samples were obtained from a local market. ENR (1000 $\mu \mathrm{g} / \mathrm{mL}$, prepared in PBS) was added to each sample for the production of spiked concentrations of $0,50,100$, and 200 $\mu \mathrm{g} / \mathrm{kg}$. Aliquots of the homogenized tissue samples (1 g of wet mass) was transferred to a $50 \mathrm{~mL}$ centrifuge tube. Exactly $5 \mathrm{~mL}$ of $5 \%$ trichloroacetic acid and $10 \mathrm{~mL}$ of $0.2 \mathrm{M}$ PBS were mixed with the tissue sample, and the mixture was incubated for $1 \mathrm{~h}$ at $60^{\circ} \mathrm{C}$. Subsequently, the suspension was centrifuged at $5000 \mathrm{~g}$ for $20 \mathrm{~min}$. The supernatant was separated and diluted tenfold with deionized water. The aliquots ( $100 \mathrm{~mL}$ each) were distributed into the microtiter plate. The ENR standards of different concentrations $(0,50,100$, and $200 \mu \mathrm{g} / \mathrm{L})$ were added to milk samples, which were then defatted by centrifugation at $5000 \mathrm{~g}$ for $20 \mathrm{~min}$ at $4{ }^{\circ} \mathrm{C}$. After $60 \mu \mathrm{L}$ of sodium nitroprusside $(0.36 \mathrm{~mol} / \mathrm{L})$ and 60 $\mu \mathrm{L}$ of zinc sulfate $(1.04 \mathrm{~mol} / \mathrm{L})$ were added to $2 \mathrm{~mL}$ of each defatted milk sample, the samples were vortexed for $1 \mathrm{~min}$ and then centrifuged at $5000 \mathrm{~g}$ for $20 \mathrm{~min}$ at $4{ }^{\circ} \mathrm{C}$. The supernatant was removed and diluted tenfold with PBS for analysis. Recoveries were calculated on the basis of the standard curve constructed by IC-ELISA. 
The specificity of the scFv under optimized conditions was evaluated by measuring cross-reactivity (CRs) with a group of structurally related compounds, including danofloxacin, ciprofloxacin, and fleroxacin. The CRs of anti-ENR scFv for ENR analogues were calculated using the formula of [CR (\%) $=I_{50}(E N R) / I C_{50}$ (ENR analogue)] $\times 100 \%$.

\section{Results}

\section{Construction of phage display ScFv library}

In this study, an ScFv library for mouse phage display was constructed, and total RNA was extracted from the spleen of immunized mice and then reverse-translated to CDNA. The VH and VL coding sequences were amplified using the cDNA as the template, and a complementary linker sequence was added. The amplified and purified NcolVL-linker and linker-VH-Not/ were spliced to whole ScFv genes through SOEPCR. As shown in Fig.1, the amplified VH, VL, and ScFv DNAs were approximately 350, 330, and 780 bp long, respectively. The purified ScFv and pCANTAB5E vectors were digested with Sfil and Notl. T4 DNA ligase was used to ligate the products, and recombinant plasmids were translated into E. coli TG1 cells, and a library with a capacity of $2.35 \times 10^{9} \mathrm{CFU} / \mathrm{mL}$ was constructed successfully.

\section{Panning of phage displayed antibody libraries}

For the production of highly specific antibodies, the washing steps were progressively increased, whereas the concentrations of coated ENR-OVA were decreased (Table 2) as described by Li et al(Cui et al., 2016). After four rounds of panning, phage-ELISA result indicated that the degree of enrichment of the specific ScFv increased gradually, as shown in Fig.2A. The size of the anti-ENR ScFv antibody library of each panning is displayed in Table 2. Ten phage-ScFvs clones were randomly collected from the fourth round's plates and identified by phage ELISA, as shown in Fig.2B. Clone ScFv-2, which showed relatively stable and high binding abilities, was selected for further study.

\section{Expression, purification of the ScFv-2}

In the preliminary experiments of this study, the highest ScFv expression level was obtained at the following conditions: $37^{\circ} \mathrm{C}, 16 \mathrm{~h}$, and $1 \mathrm{mM} \mathrm{IPTG}$. Under optimal expression conditions, the ScFv-2 antibody fragments were expressed in E. coli HB2151, and proteins were determined through Western blotting, as shown in Fig.3. 3.3.

\section{IC-ELISA for ENR and its analogues based on ScFv-2}

The performance of the purified ScFv-2 was evaluated using IC-ELISA. The optimum concentration of ScFv-2 was $0.25 \mu \mathrm{g} / \mathrm{mL}$, producing an $\mathrm{OD}_{450}$ of 1.0 at $4 \mu \mathrm{g} / \mathrm{mL}$ of ENR-OVA coating concentration through checkerboard titration. Under the optimal conditions, the regression curve equation of ENR-ScFv was $y=-0.4369 x+1.0788\left(R^{2}=0.9911\right)$, as shown in Fig.6. The $I_{50}$ value of the assay established with 
ScFv-2 was $21.08 \mathrm{ng} / \mathrm{mL}$, demonstrating that ScFv can be used in detecting ENR. The linear range of the assay established with ScFv ranged from $4.34 \mathrm{ng} / \mathrm{mL}$ to $173.90 \mathrm{ng} / \mathrm{mL}$.

\section{Homology modeling and molecular docking}

In this experiment, given the results of parameter evaluation, such as sequence identity and structural similarity, the protein PDB ID: 5B3N was selected as the template sequence, which has the highest sequence identity $(68 \%)$ with the target sequence anti-ENR-ScFv. Subsequently, a stable model of singlechain antibody was established using the SWISS MODEL homology modeling software. The single-chain antibody of anti-ENR-ScFv was connected by VH and VL through three connecting peptides (Gly4Ser) and has a typical single-chain antibody structure with anti-parallel $\beta$-sheet and loop regions.

The docking result of ScFv-2 with ENR is shown in Fig.5A. The active site of ScFv-2 consists of residues Ser46, Val48, Leu56, Trp58, Ser61, Asp109, Tyr119, Leu121, Leu153, Cys235, Leu236, Gln237, Tyr238, Asp239, Glu240, Phe241, and Pro242. The carboxyl group of ENR can form a $2.2 \AA$ hydrogen bond with the residue Asp109. The quinoline structure is located in the hydrophobic cavity formed by residues Val48, Phe241, Leu236, and Tyr238. The cyclopropyl group can interact with the residues Phe241 and Leu236 and forms hydrophobic interaction with Tyr238. Thus, these forces may be the main reason for the increased ability to bind to ENR. However, the hydrophilic carboxyl group in ENR and the carbonyl group on the quinoline are close to the hydrophobic residue Leu121. We preliminarily speculated that if the residue Leu121 is transformed to a hydrophilic amino acid, it will promote the combination of ENR to ScFv. When Leu121 was substituted by Asn, the total binding energy decreased from -5.09 to -7.83 $\mathrm{kcal} / \mathrm{mol}$, and the number of hydrogen bonds and the amino acids forming hydrophobic interaction all increased in the binding site (Fig.5B and 5C), indicating that the intermolecular forces of ScFv-ENR increased. Therefore, Leu121 was substituted with Asn for the directional mutagenesis of the ScFv antibody in the present study.

\section{Characterization of mutant ScFv}

The performance of purified ScFv mutant was evaluated through SDS-PAGE and IC-ELISA. Under the same conditions as the parental ScFv, the regression curve equation of the mutant ScFv was $y=-0.192 x+0.5423\left(R^{2}=0.9994\right)$, as shown in Fig.6. The $\mathrm{IC}_{50}$ value of the assay established with the ScFv mutant was $1.63 \mathrm{ng} / \mathrm{mL}$, indicating that the affinity of the ScFv mutant increased 12.9 times compared with that of the parental ScFv. The mutant had a higher affinity and better sensitivity than the original antibody, indicating that the parental ScFv antibody was evolved successfully.

\section{Precision and recovery}

Spiking and recovery tests were conducted for the assessment of the feasibility of IC-ELISA. During the tests, the mutant scFv was used in detecting ENR in spiked samples, and no positive results were obtained for the non-spiked samples. All samples spiked with ENR showed good agreement between the spiking level and concentration detected, as shown in Table 3. In the intra-assay, the mean recovery for 
ENR ranged from $71.80 \%$ to $114.58 \%$ and the RSD values ranged between $1.81 \%$ and $7.65 \%$ (based on triple measurements within a day). In the inter-assay, the mean recovery for ENR ranged from $70.29 \%$ to $117.35 \%$, and RSD values ranged between $1.53 \%$ and $10.46 \%$ (based on triple measurements in 3 days). As demonstrated with the samples spiked with ENR, the IC-ELISA method provided satisfactory results for the detection of ENR residues in milk and food animal tissues. The cross-reactivity of the ENR-scFv with danofloxacin, ciprofloxacin, and fleroxacin and many more were tested through IC-ELISA. As shown in Table 4, the ENR-scFv showed low cross-reactivity with other fluoroquinolones, indicating that scFv is highly specific for ENR.

\section{Discussion}

ScFv is a synthetic antibody with only one chain and has a specific affinity for antigens. Compared with conventional antibodies (Mabs and Pabs), the ScFv antibody can be studied at the molecular level (homologous modeling and molecular docking), and its antigen binding affinity can be improved through gene mutation and gene reforming (Liu et al., 2016). In addition, the antibody can be produced on a large scale in prokaryotic and eukaryotic systems and is thus cheap and time saving.(Chaisri \& Chaicumpa, 2018; X. Zhang, Zhang, Liu, Yu, \& Liu, 2010).

As is well known, IPTG concentration, post-induction time, and incubation temperature are the main factors for optimizing protein expression (Dong, Bo, Zhang, Feng, \& Liu, 2018).

The results indicated that the ScFv-2 antibody (approximately $47 \mathrm{kDa}$ ) was expressed successfully. AntiENR ScFv proteins were purified using Beaver Beads ${ }^{T M}$ His-tag Protein Purification magnetic beads according to the manufacturer's instructions. The purity of protein solution was confirmed through SDSPAGE, as shown in Fig.4, then the purified protein was stored at $-20^{\circ} \mathrm{C}$.

We evaluated the model to verify its reliability. The Procheck program was used in evaluating the threedimensional structure of the anti-ENR-ScFv model. Ramachandran plot showed that $91.1 \%$ of the amino acids residues in the model were located in the core region, $7.9 \%$ in the allowable region, and only $1 \%$ in the forbidden zone of the twist angle. The results showed that the dihedral angles of $99 \%$ protein residues in ScFv model were within the reasonable range and conformed to the rule of stereochemical energy. The result of Verify-3D showed that the average 3D-1D score of $92.2 \%$ amino acid residues in the ScFv model was greater than 0.2 , and the model passed the verify3d test. The Errat result showed that the overall quality factor was 98.445 . Therefore, the experimental model of anti-ENR-ScFv has high reliability and can be used in the molecular docking of the ENR antigen.

A highly sensitive anti-ENR single-chain antibody was obtained through phage display and directional evolution, and a rapid and highly sensitive IC-ELISA method for detecting ENR residues in products of animal origin was developed. The method showed good stability, reproducibility, and accuracy for detecting ENR, indicating a wide application prospect for the rapid and sensitive detection of antibiotic residues. 


\section{Abbreviations}

SCFv, single-chain antibody; IC-ELISA, indirect competitive enzyme-linked immunosorbent assay; ENR, Enrofloxacin; FQs, fluoroquinolones; MRL, maximum residue limits; MAbs, monoclonal antibodies; VH, variable-heavy chain; VL, variable light chain; SAR, sarafloxacin; DIF, difloxacin; CIP, ciprofloxacin; LOM, Lomefloxacin; ENO, enofloxacin; NOR, norfloxacin; PEF, pefloxacin; SOE-PCR, splicing overlap extension PCR;LOD, limit of detection; CRs, cross-reactivity.

\section{Declarations}

\section{Ethics approval and consent to participate}

This article does not contain any studies with human participants or animals performed by any of the authors.

\section{Consent for publication}

All authors consent for the manuscript to be published.

\section{Availability of data and material}

All data generated or analysed during this study are included in this article.

\section{Competing interests}

The authors declare that they have no competing interests.

\section{Authorship contribution statement}

Fang-yu Wang: Conceptualization, Methodology, Investigation, Writing - original draft, Visualization, Funding acquisition, Supervision and Project administration. Ning Li and Yun-shang Zhang: Methodology, Formal analysis, Investigation, Visualization. Guang-xu Xing: Conceptualization, Methodology, Resources. Jian-ming Fan囚Review \& editing.

\section{Declaration of Competing Interest}

The authors declare that they have no known competing financial interests or personal relationships that could have appeared to influence the work reported in this paper.

\section{Acknowledgements}

This research was supported by The National Key Research and Development Program of China (No. 2018YFC1602902区.

\section{References}


Abdelwahab, M., Loa, C. C., Wu, C. C., \& Lin, T. L. (2015). Recombinant nucleocapsid protein-based enzyme-linked immunosorbent assay for detection of antibody to turkey coronavirus. Journal of virological methods, 217, 36-41.

Bird, S. T., Etminan, M., Brophy, J. M., Hartzema, A. G., \& Delaney, J. A. C. (2013). Risk of acute kidney injury associated with the use of fluoroquinolones. CMAJ : Canadian Medical Association journal = journal de l'Association medicale canadienne, 185 (10), E475-E482.

Chaisri, U., \& Chaicumpa, W. (2018). Evolution of Therapeutic Antibodies, Influenza Virus Biology, Influenza, and Influenza Immunotherapy. BioMed research international, 2018, 9747549-9747549.

Cui, L., Jinxin, H., Hao, R., Xiaoying, Z., Enqi, D., \& Xinping, L. (2016). Preparation of a Chicken scFv to Analyze Gentamicin Residue in Animal Derived Food Products. Analytical chemistry, 88 (7).

Dalhoff, A. (2015). Antiviral, antifungal, and antiparasitic activities of fluoroquinolones optimized for treatment of bacterial infections: a puzzling paradox or a logical consequence of their mode of action? European journal of clinical microbiology \& infectious diseases : official publication of the European Society of Clinical Microbiology, 34 (4), 661-668.

Dong, S., Bo, Z., Zhang, C., Feng, J., \& Liu, X. (2018). Screening for single-chain variable fragment antibodies against multiple Cry1 toxins from an immunized mouse phage display antibody library. App/ Microbiol Biotechnol, 102 (7), 3363-3374.

Fan, G.-y., Yang, R.-s., Jiang, J.-q., Chang, X.-y., Chen, J.-j., Qi, Y.-h., Wu, S.-x., \& Yang, X.-f. (2012). Development of a class-specific polyclonal antibody-based indirect competitive ELISA for detecting fluoroquinolone residues in milk. Journal of Zhejiang University. Science. B, 13 (7), 545-554.

Huang, B., Yin, Y., Lu, L., Ding, H., Wang, L., Yu, T., Zhu, J.-j., Zheng, X.-d., \& Zhang, Y.-z. (2010). Preparation of high-affinity rabbit monoclonal antibodies for ciprofloxacin and development of an indirect competitive ELISA for residues in milk. Journal of Zhejiang University. Science. B, 11 (10), 812-818.

Jing, L., C, Z. H., F, D. C., Jun, D., X, Z. G., P, W. J., Nan, L., Z, L. J., \& W, L. Y. (2016). Production of antiamoxicillin ScFv antibody and simulation studying its molecular recognition mechanism for penicillins. Journal of environmental science and health. Part. B, Pesticides, food contaminants, and agricultural wastes, 51 (11).

Kang, J., Hossain, M. A., Park, H.-C., Kim, Y., Lee, K.-J., \& Park, S.-W. (2019). Pharmacokinetic and pharmacodynamic integration of enrofloxacin against Salmonella Enteritidis after administering to broiler chicken by per-oral and intravenous routes. Journal of veterinary science, 20 (2), e15-e15.

Kumari, M., \& Kumar, A. (2020). Human health risk assessment of antibiotics in binary mixtures for finished drinking water. Chemosphere, 240. 
Li, C., Luo, X., Li, Y., Yang, H., Liang, X., Wen, K., Cao, Y., Li, C., Wang, W., Shi, W., Zhang, S., Yu, X., \& Wang, Z. (2019). A Class-Selective Immunoassay for Sulfonamides Residue Detection in Milk Using a Superior Polyclonal Antibody with Broad Specificity and Highly Uniform Affinity. Molecules (Basel, Switzerland), 24 (3), 443.

Li, J., Hao, H., Cheng, G., Wang, X., Ahmed, S., Shabbir, M. A. B., Liu, Z., Dai, M., \& Yuan, Z. (2017). The effects of different enrofloxacin dosages on clinical efficacy and resistance development in chickens experimentally infected with Salmonella Typhimurium. Scientific reports, 7(1), 11676-11676.

Lin, Y.-W., Yu, H. H., Zhao, J., Han, M.-L., Zhu, Y., Akter, J., Wickremasinghe, H., Walpola, H., Wirth, V., Rao, G. G., Forrest, A., Velkov, T., \& Li, J. (2018). Polymyxin B in Combination with Enrofloxacin Exerts Synergistic Killing against Extensively Drug-Resistant Pseudomonas aeruginosa. Antimicrobial agents and chemotherapy, 62 (6), e00028-00018.

Liu, J., Zhang, H. C., Duan, C. F., Dong, J., Zhao, G. X., Wang, J. P., Li, N., Liu, J. Z., \& Li, Y. W. (2016). Production of anti-amoxicillin ScFv antibody and simulation studying its molecular recognition mechanism for penicillins. Journal of Environmental Ence \& Health.part.b Pesticides Food Contaminants \& Agricultural Wastes, 742.

Makvandi-Nejad, S., Sheedy, C., Veldhuis, L., Richard, G., \& Hall, J. C. (2010). Selection of single chain variable fragment (scFv) antibodies from a hyperimmunized phage display library for the detection of the antibiotic monensin. Journal of Immunological Methods, 360 (1-2), 103-118.

Norihiro, K. (2008). Anti-estradiol-17beta single-chain Fv fragments: Generation, characterization, gene randomization, and optimized phage display. Steroids, 14 (73).

Norihiro, K. (2010). Two-step in vitro antibody affinity maturation enables estradiol-17beta assays with more than 10-fold higher sensitivity. Analytical chemistry, 3 (82).

Patel, K., \& Goldman, J. L. (2016). Safety Concerns Surrounding Quinolone Use in Children. Journal of clinical pharmacology, 56 (9), 1060-1075.

Tao, X., Chen, M., Jiang, H., Shen, J., Wang, Z., Wang, X., Wu, X., \& Wen, K. (2013). Chemiluminescence competitive indirect enzyme immunoassay for 20 fluoroquinolone residues in fish and shrimp based on a single-chain variable fragment. Analytical and Bioanalytical Chemistry, 405 (23), 7477-7484.

Wang, J. P., Dong, J., Duan, C. F., Zhang, H. C., He, X., Wang, G. N., Zhao, G. X., \& Liu, J. (2016). Production and Directional Evolution of Antisarafloxacin ScFv Antibody for Immunoassay of Fluoroquinolones in Milk. J Agric Food Chem, 64 (42), 7957-7965.

Wen, K., Nolke, G., Schillberg, S., Wang, Z., Zhang, S., Wu, C., Jiang, H., Meng, H., \& Shen, J. (2012). Improved fluoroquinolone detection in ELISA through engineering of a broad-specific single-chain variable fragment binding simultaneously to 20 fluoroquinolones. Anal Bioanal Chem, 403 (9), 2771-2783. 
Xu, L., Wang, H., Yang, X., \& Lu, L. (2013). Integrated pharmacokinetics/pharmacodynamics parametersbased dosing guidelines of enrofloxacin in grass carp Ctenopharyngodon idella to minimize selection of drug resistance. BMC veterinary research, 9, 126-126.

Zhang, H.-t., Jiang, J.-q., Wang, Z.-I., Chang, X.-y., Liu, X.-y., Wang, S.-h., Zhao, K., \& Chen, J.-s. (2011). Development of an indirect competitive ELISA for simultaneous detection of enrofloxacin and ciprofloxacin. Journal of Zhejiang University. Science. B, 12 (11), 884-891.

Zhang, X., Zhang, C., Liu, Y., Yu, X., \& Liu, X. (2010). Construction of scFv phage display library with hapten-specific repertories and characterization of anti-ivermectin fragment isolated from the library. European Food Research and Technology, 231 (3).

\section{Tables}

Table 1 Nucleotide primer sequences.

\begin{tabular}{|ll|}
\hline Primer names & Nucleotide Sequences $\left(\mathbf{5}^{\prime} \rightarrow \mathbf{3}^{\prime}\right)$ \\
\hline VH for & GCGGCCCAGCCGGCCATGGCCGARGTGAAGCTGGTGGARTCTGGR \\
\hline VH back & AGCGGCGGTGGCGGTTCTGGAGGCGGCGGTTCTGAYATGCAGATGACMCAG \\
\hline VL for & AGCGGCGGTGGCGGTTCTGGAGGCGGCGGTTCTRAMATTGTGMTGACCCAATC \\
& AGCGGCGGTGGCGGTTCTGGAGGCGGCGGTTCTGAYATGCAGATGACMCAGWC \\
\hline VL back & ACTAGTCGCGGCCGCGTCGACAGCMCGTTTBAKYTCTATCTTTGT \\
\hline scFv for & CGCAATTCCTTTAGTTGTTCCTTTCTATGCGGCCCAGCCGGCCATGGCC \\
\hline scFv back & GGTTCCAGCGGATCCGGATACGGCACCGGACTAGTCGCGGCCGCGTCGAC \\
\hline
\end{tabular}

Table 2 Enrichment of phage display librar. 


\begin{tabular}{|llllll|}
\hline rounds & coated antigen & Coating & input & output & output/input \\
\hline 1 & ENR-OVA & $10 \mu \mathrm{g}$ & $4 \times 10^{11}$ & $3.1 \times 10^{5}$ & $7.75 \times 10^{-7}$ \\
\hline 2 & ENR-OVA & $10 \mu \mathrm{g}$ & $4 \times 10^{11}$ & $5.8 \times 10^{6}$ & $1.45 \times 10^{-5}$ \\
\hline 3 & ENR-OVA & $10 \mu \mathrm{g}$ & $4 \times 10^{11}$ & $2.6 \times 10^{8}$ & $6.5 \times 10^{-4}$ \\
4 & ENR-OVA & $5 \mu \mathrm{g}$ & $4 \times 10^{11}$ & $2.35 \times 10^{9}$ & $5.87 \times 10^{-3}$ \\
\hline
\end{tabular}

Table 3 Recovery of enr from fresh milk, pork, beef and chicken samples $(n=3)$.

\begin{tabular}{|c|c|c|c|c|c|c|c|}
\hline \multirow[t]{2}{*}{ Samples } & \multirow[t]{2}{*}{ Spiked } & \multicolumn{3}{|c|}{ Intra-assay } & \multicolumn{3}{|c|}{ Inter-assay } \\
\hline & & Found ${ }^{a}$ & Recovery (\%) & RSDr (\%) & Found ${ }^{b}$ & Recovery (\%) & RSDr (\%) \\
\hline \multirow[t]{4}{*}{ Milk } & 0 & $<L O D$ & - & -- & $<\angle O D$ & - & - \\
\hline & 50 & 39.487 & 78.97 & 5.67 & 39.051 & 78.10 & 6.73 \\
\hline & 100 & 71.805 & 71.80 & 5.54 & 90.896 & 90.89 & 10.46 \\
\hline & 200 & 179.704 & 89.85 & 4.95 & 180.792 & 90.39 & 10.34 \\
\hline \multirow[t]{4}{*}{ Pork } & 0 & $<\mathrm{LOD}$ & - & - & $<\angle O D$ & - & -- \\
\hline & 50 & 40.569 & 81.13 & 4.52 & 35.149 & 70.29 & 2.49 \\
\hline & 100 & 79.607 & 79.60 & 5.16 & 90.167 & 90.167 & 5.33 \\
\hline & 200 & 229.174 & 114.58 & 7.65 & 193.531 & 96.76 & 7.81 \\
\hline \multirow[t]{4}{*}{ Beef } & 0 & $<\mathrm{LOD}$ & - & - & $<L O D$ & - & - \\
\hline & 50 & 41.563 & 83.12 & 3.11 & 39.617 & 79.23 & 2.31 \\
\hline & 100 & 87.315 & 87.31 & 2.45 & 90.497 & 90.49 & 4.90 \\
\hline & 200 & 195.89 & 97.94 & 1.81 & 189.896 & 94.94 & 5.49 \\
\hline \multirow[t]{4}{*}{ Chicken } & 0 & $<L O D$ & -- & - & $<L O D$ & - & -- \\
\hline & 50 & 53.607 & 107.21 & 5.17 & 48.071 & 96.14 & 1.59 \\
\hline & 100 & 90.362 & 90.36 & 5.82 & 81.531 & 81.53 & 1.53 \\
\hline & 200 & 196.162 & 98.08 & 6.49 & 234.708 & 117.35 & 2.97 \\
\hline
\end{tabular}


a: The spiked concentrations are in units of $\mu \mathrm{g} / \mathrm{kg}$ or $\mu \mathrm{L} / \mathrm{kg}$; b: the found concentrations are in units of $\mu \mathrm{g} / \mathrm{kg}$ or $\mu \mathrm{L} / \mathrm{kg}$; RSDr: relative standard deviation under repeatability conditions; RSDR: intralaboratory reproducibility conditions.

Table 4 Cross-reactivity of scfv mutant with various fluoroquinolones antibiotics.

\begin{tabular}{|ll|}
\hline Analytes & Cross-reactivity (\%) \\
\hline Enrofloxacin & 100 \\
\hline Danofloxacin & 10 \\
\hline Ciprofloxacin & 6 \\
\hline Fleroxacin & 9 \\
Sarafloxacin & 11 \\
Norfloxacin & 24 \\
Difloxacin & 16 \\
Pefloxacin & 21 \\
Amifloxacin & 30 \\
Lomefloxacin & 23 \\
Enoxacin & 4 \\
\hline
\end{tabular}

\section{Figures}



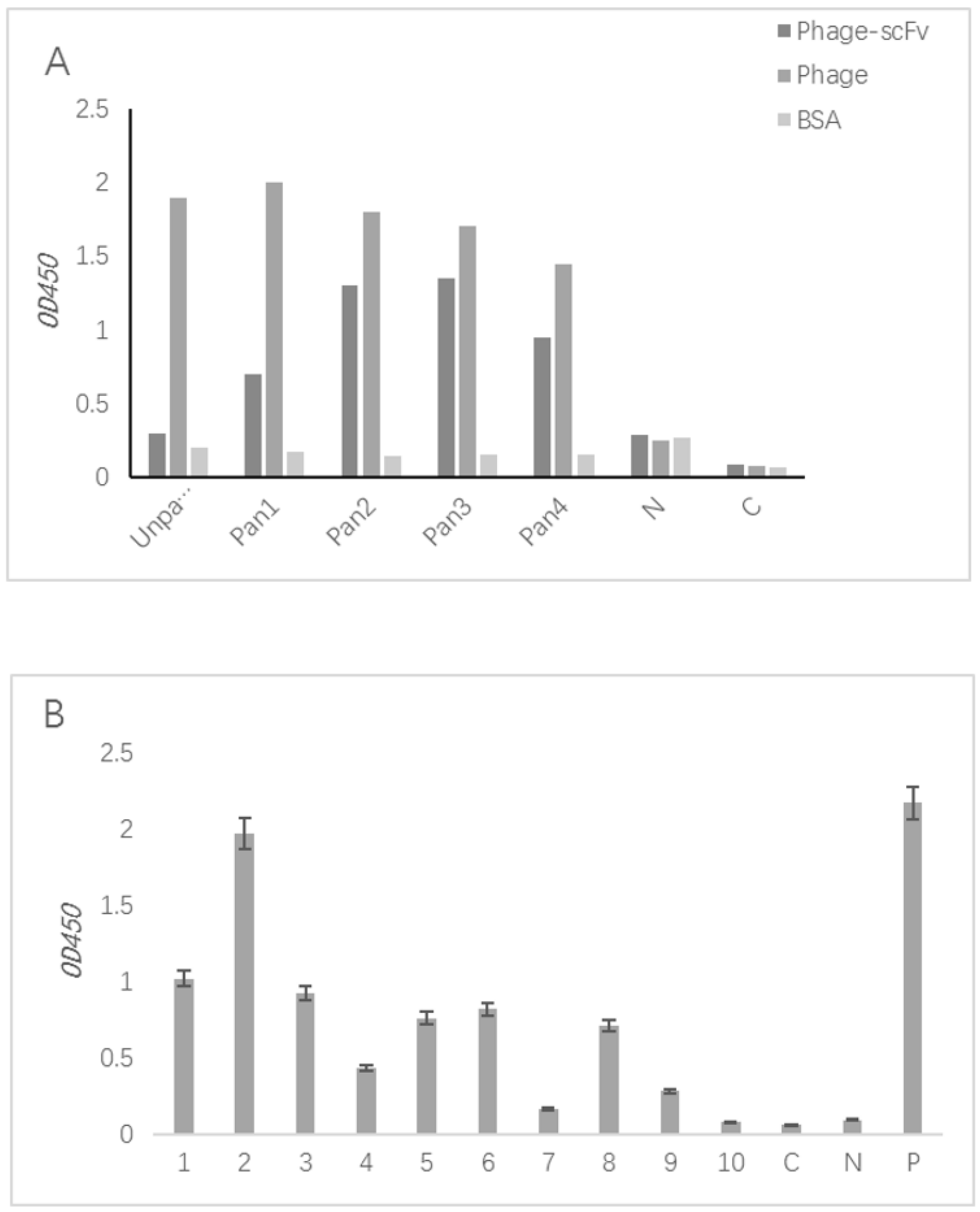

\section{Figure 1}

Phage-ELISA. (A) The enrichment of specific scFv in each library after four rounds panning. (B) Binding activity of ScFv antibodies to ENR. 1-10: ScFv antibodies from randomly selected clones from the 4th panning; C: blank control; N: negative control, BSA; P: positive control, cell supernatant. 
A

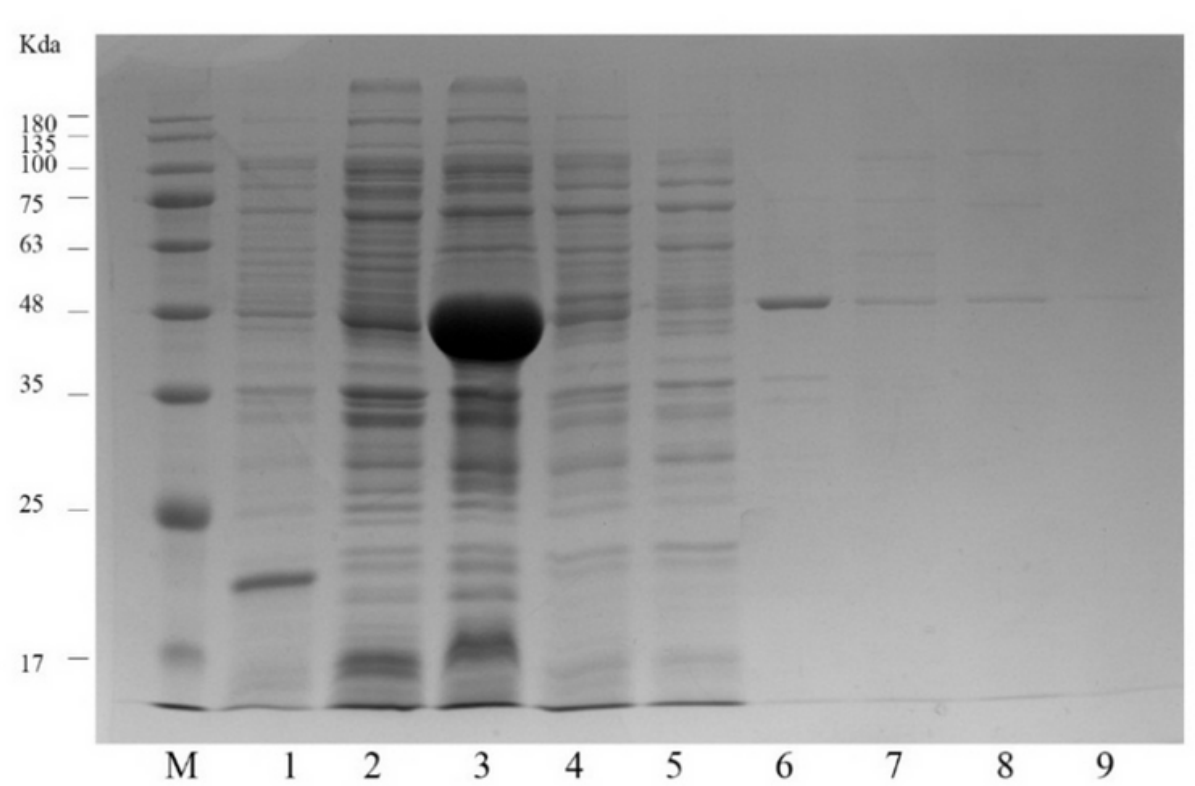

B

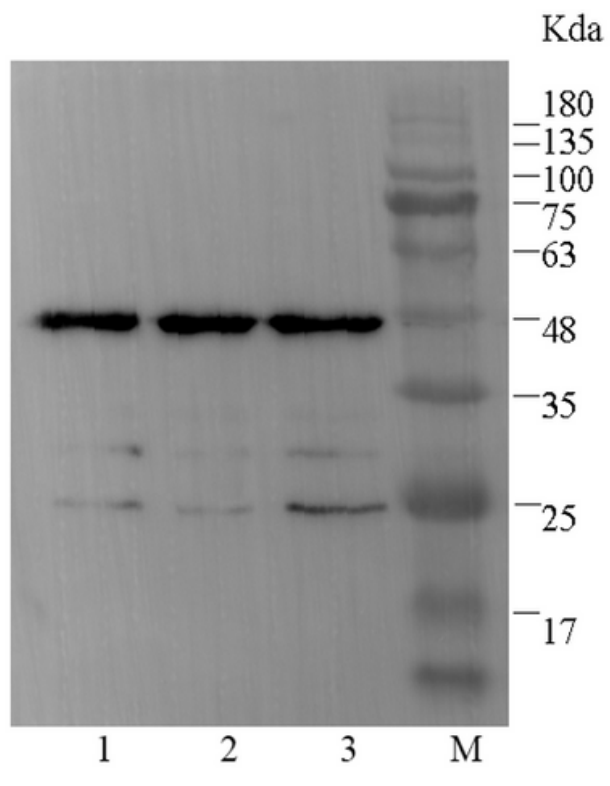

Figure 2

Analysis of ScFv-2 by Western blot. Lane 1-3: ScFv-2 induced expression. Lane M: Protein 180 marker. 
A
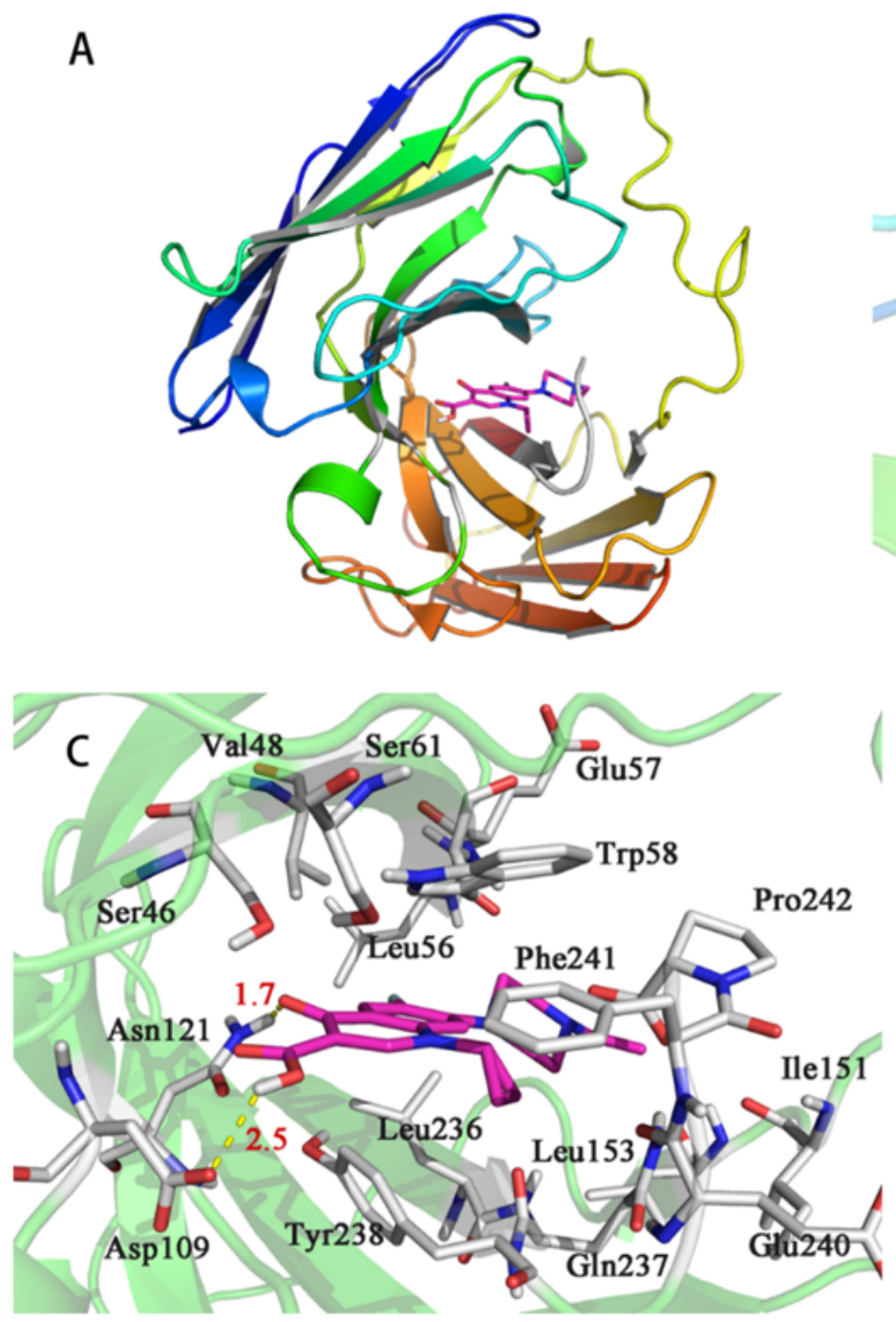
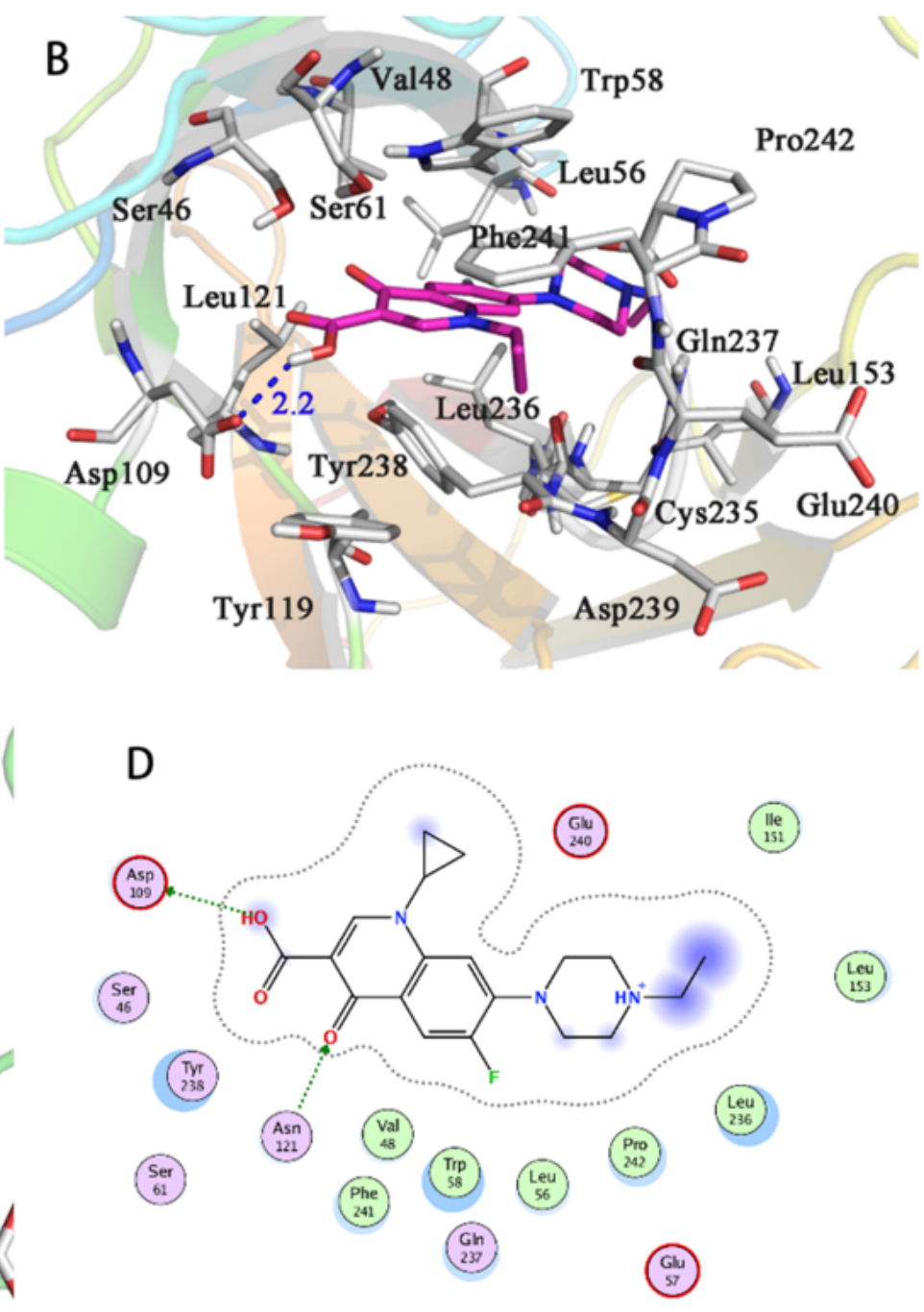

Figure 3

A: The binding mode between parental ScFv and ENR, B: The interaction between parental ScFv and ENR binding site; $\mathrm{C}$ : The interaction between ScFv mutant and ENR binding site. 


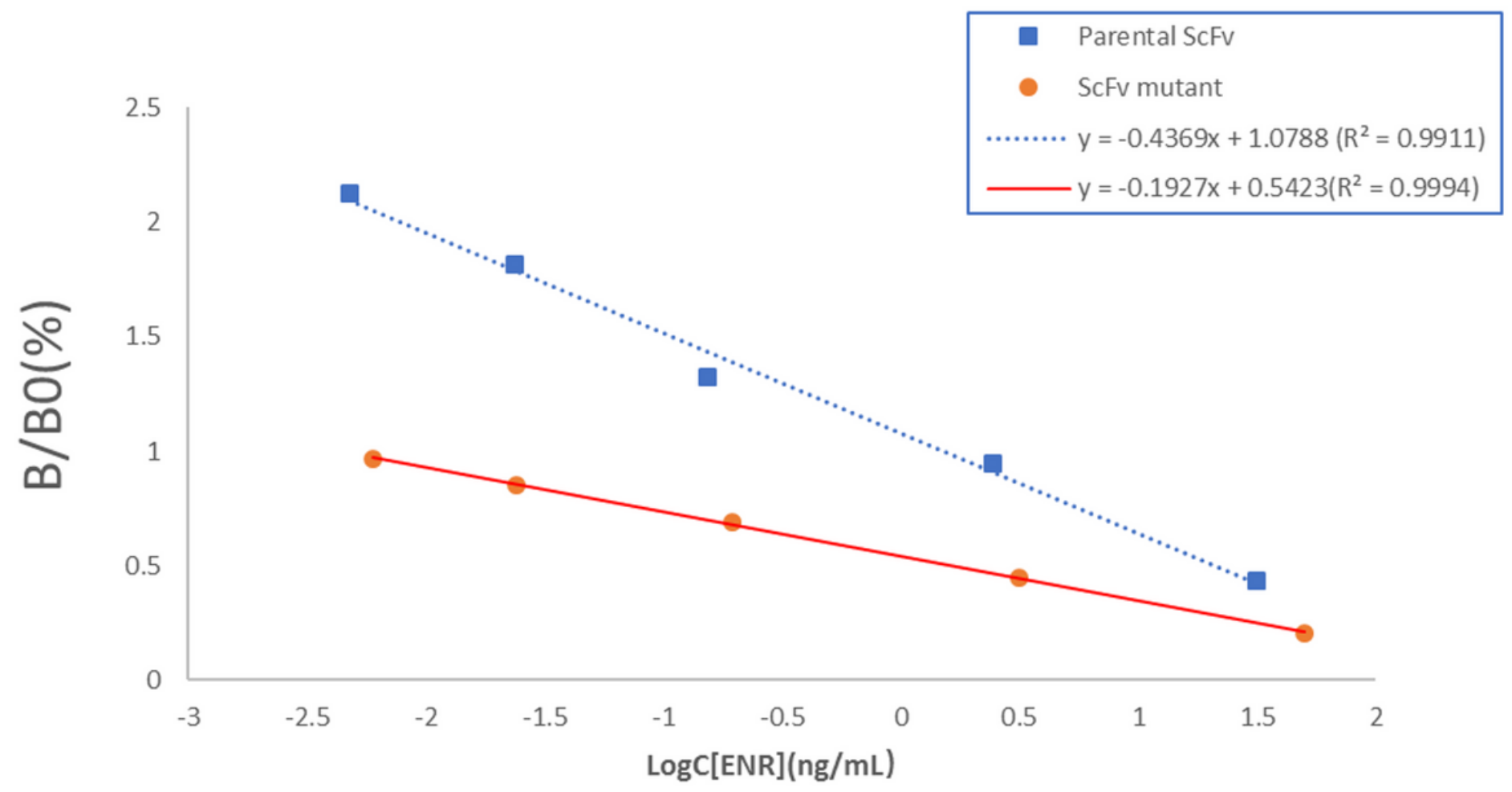

Figure 4

Standard curves of the competitive ELISA for ENR. X-axis logarithm concentration of ENR, Y-axis represents the inhibition rate $(\mathrm{B} / \mathrm{B} 0)$. $\mathrm{B} 0$ and $\mathrm{B}$ are the absorbance values obtained from binding at zero and certain concentrations of ENR standard. 